\title{
Application of CAD-based Traditional Patterns in Interior Design
}

\author{
Hongmei Jia $^{1 \mathrm{a}}$, Siyuan Kuai ${ }^{2 \mathrm{~b}}$, Jiali Dong ${ }^{3 \mathrm{c}}$ \\ ${ }^{1}$ academy of arts, Jiangsu University Zhenjiang, Jiangsu, China \\ ${ }^{2}$ academy of arts, Jiangsu University Zhenjiang, Jiangsu, China \\ ${ }^{3}$ academy of arts, Jiangsu University Zhenjiang, Jiangsu, China
}

\begin{abstract}
Starting with the approach of representing traditional patterns with CAD in interior design, this paper analyzes the scenarios of application of traditional patterns realized through graphics, images and models and sets out that it is essential to analyze basic laws and characteristics of patterns as well as their fixed and variable elements and apply them appropriately to the interior space in order to achieve the purpose. The analysis on variable elements of traditional patterns is carried out from three aspects, namely the appropriate embellishment of colored painting, direct application of traditional partition boards and innovation based on traditional patterns.
\end{abstract}

\section{INTRODUCTION}

Many designers have been experimenting with Chinesestyle architectural interior forms. They usually process traditional symbols and reflect the connotation of traditional Chinese culture with abstract or simplified techniques. What we should do is to incorporate as many beautiful traditional Chinese patterns as possible and design interior decorative styles conforming with the aesthetics of the Chinese people based on full understanding of the modern architectural style. The use of CAD for interior design is common in the industry but shallow in the application to traditional patterns. Real traditional elements are cited and displayed in the indoor environment directly without any deformation or embellishment, such as window carvings, Ming Dynasty furniture and screens with traditional patterns. Traditional patterns in interior design should be combined with the architectural structure. This paper attempts to identify the problems to be solved in the application of traditional patterns to interior design from the perspective of CAD.

\section{GRAPHICS CLASSIFICATION OF CAD OF TRADITIONAL PATTERNS}

In the interior design, traditional patterns are imaged and presented through CAD in three categories based on the difference in technical approaches:

\subsection{Graphics}

The virtual effect of interior design is manifested by graphics obtained from computer mapping. The graphics involved are generally considered as those abstracted from objects in the objective world, which contain the color and shape information. They are two-dimensional and have the most basic graphic elements including points, lines, circles and arcs. Their information includes the geometric and attribute information of primitives, such as the color, line type and width and other explicit attributes as well as the layer and other implicit attributes. Pictures of traditional patterns are generally referred to for lofting of such graphics. The graphics produced are the basis of images and models and engineering drawings that can be referred to for indoor construction. They are applied to the surbase of indoor column and beam frame structures and as the sketch of decorative patterns and the line graph source for structural modeling.

\subsection{Images}

Images are graphics expressed by dot matrices with color information in the computer, which record grayscale or color elements. They may be photos, scanned pictures and graphics generated by a computer. The geometric position and display attributes of images are determined as needed and manifested on the computer through static images or dynamic pictures. In the interior space, such graphics are mainly used for interface decoration and the surface decoration of beam frames, partitions and other structures.

\subsection{Models}

Models combine various vector graphics, especially manifest the geometry into three-dimensional stereograms and focuses on the representation of indoor structure. In terms of the application scenarios of traditional patterns, line graphs are mainly subjected to secondary processing to form an architectural or display structure more adapted to the space and finally produce a complete and vivid 
interior space picture with the material, color and mechanism attached. Examples are windows, doors, partitions, beam frames and furnishings with traditional patterns.

\section{ESSENTIALS OF CAD OF TRADITIONAL PATTERNS IN INTERIOR SPACE}

In the design of of interior space of traditional styles, whether graphics, images or models, it is necessary to study traditional patterns in depth to understand their basic laws and characteristics as well as their fixed and variable elements and apply them appropriately to interior space. Let's take the beam and square-column colored painting in the Ming and Qing Dynasties for example. It has a set pattern and cannot be simply scaled to adapt to the indoor column spacing, in which case the harmony of the original colored painting will be lost. The beam and square-column colored painting of buildings in the Palace Museum is a model for such application as shown in Figure 1. There are no shortage of excellent cases of deformation and re-creation in modern architecture (Figure 2).

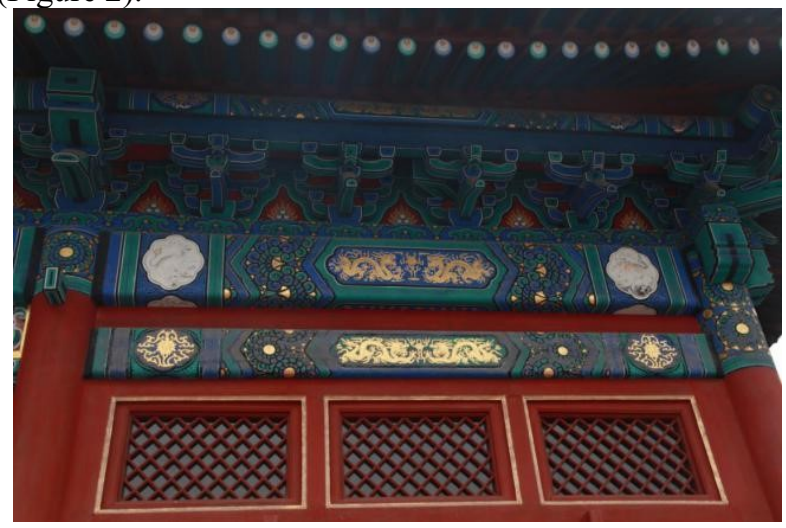

Figure 1 Xuanzi beam and square-column colored painting in the Palace Museum, Beijing (photographed by the author)

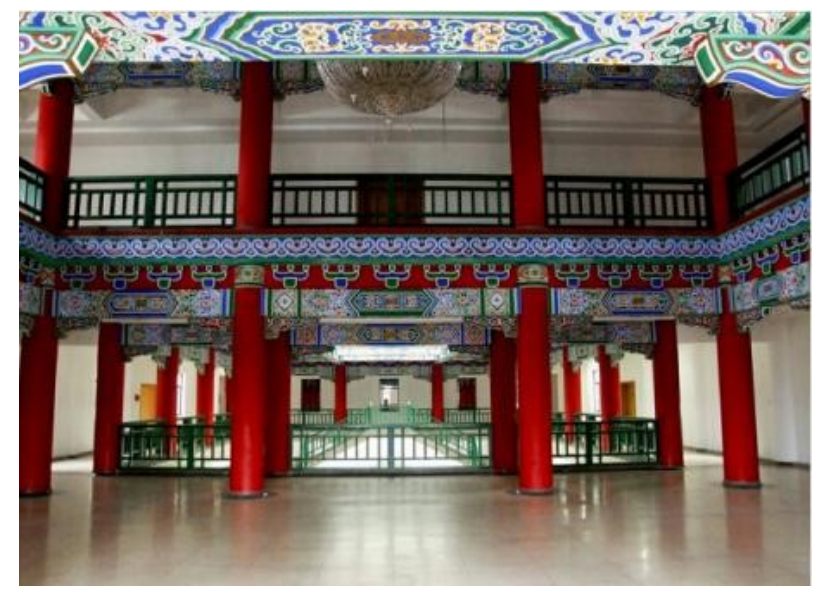

Figure 2 Interior of Shanghai Library (photographed by the author)

It is required to get aware of the generation, inherent application scenarios, materials and processing methods and implicit cultural significance of traditional patterns used in the interior space based on their different features, analyze their fixed and variable elements, especially the latter, and then determine the extent of their deformation or embellishment for application to an indoor environment, in the case of both the creation of vector graphics to form images (e.g. creating graphic lines with AUTOCAD and filling colors with PHOTOSHOP to form images, or creating images directly with PHOTOSHOP and other image processing software) and sources of interior maps and the 3D modelling with modelling software.

\section{VARIABLE ELEMENTS OF TRADITIONAL PATTERNS}

This paper analyzes fixed and variable elements of traditional patterns in three aspects based on their different application characteristics.

\subsection{Appropriate embellishment of colored painting}

The basic function of colored painting was originally timber protection to extend its life, and decoration was an incidental result. However, the ancients were good at colored painting and used it to express the structural spirit of buildings. The colored painting system in different eras differed due to the difference in its structural approach. As the beam and square-column colored painting in the Ming and Qing Dynasties has developed a set pattern, it is easier to carry out computer-aided design and it has more extensive scenarios of application in the interior space.

The beam and square-column colored painting in the Ming and Qing Dynasties is divided into three categories by grade, namely Hexi, Xuanzi and Sushi. Its basic structure is roughly composed of three parts - the central portion (a), end portions (c) and intermediate portions (b) which are roughly equal in length as shown in Figure 3 Structure of Xuanzi colored painting. Let's take the Xuanzi colored painting in the Qing Dynasty for example. Convolvulus tricolor patterns within intermediate portions are divided into several parts as shown in Figure 4 based on the difference in width and layout. Patterns in intermediate portions are adaptive variables. In the case of changes in column spacing in the application to interior design, the method of transverse stretching in the same proportion is generally not used; instead, patterns within intermediate portions are changed to adapt to the overall length, which will not damage the complete form of patterns in the central portion as shown in Figure 1.

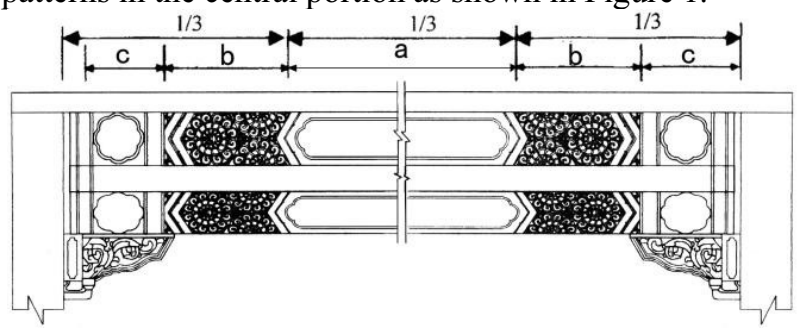

Figure 3 Structure of Xuanzi colored painting (drawn by the author) 


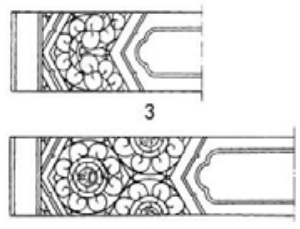

6

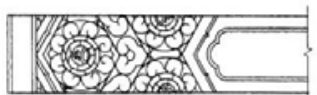

7.5

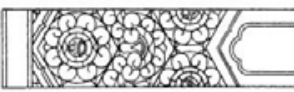

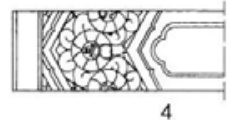
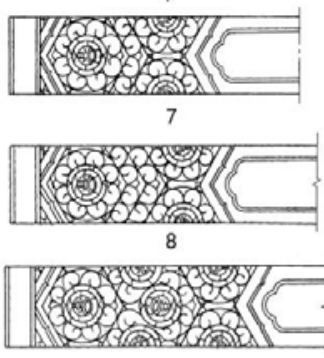

Figure 4 Convolvulus tricolor patterns and width (drawn by the author)

\subsection{Direct application of traditional partition boards}

Designers apply traditional patterns directly to the interior interface. For example, traditional partition boards are used for the partition of indoor and outdoor space. In the technique, real traditional elements are cited and displayed directly in the indoor environment without excessive deformation and embellishment, such as window carvings, Ming Dynasty furniture and screens with traditional patterns, to strengthen the experience of people in Chinese classical culture and its appeal. Traditional partition boards have substantially fixed forms, mainly including partition windows and doors. Partition doors can be designed and selected in an adaptive way based on the interior height. Figure 5 shows the structure of a partition door composed of the frame, the heart, sash ring plates and skirts. The height of skirts can be increased slightly based on the difference in the indoor height. The best way is to increase the number of sash ring plates and window stools, thus retaining the longtime fine proportional relation of all parts of traditional window sashes as shown in Figure 6

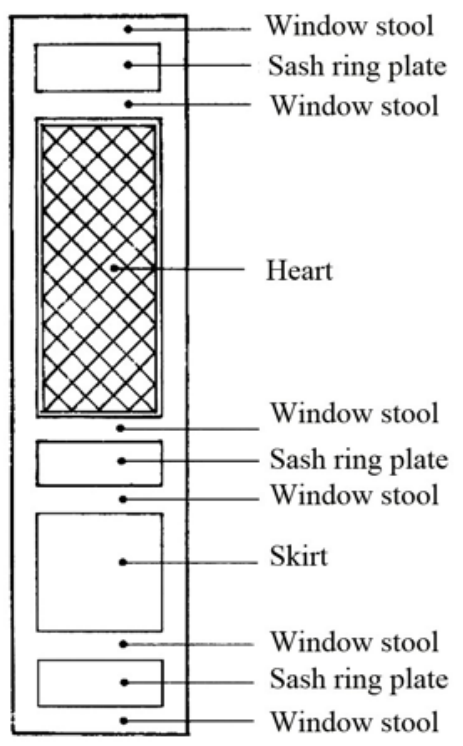

Figure 5 Basic composition of partition boards (drawn by the author)
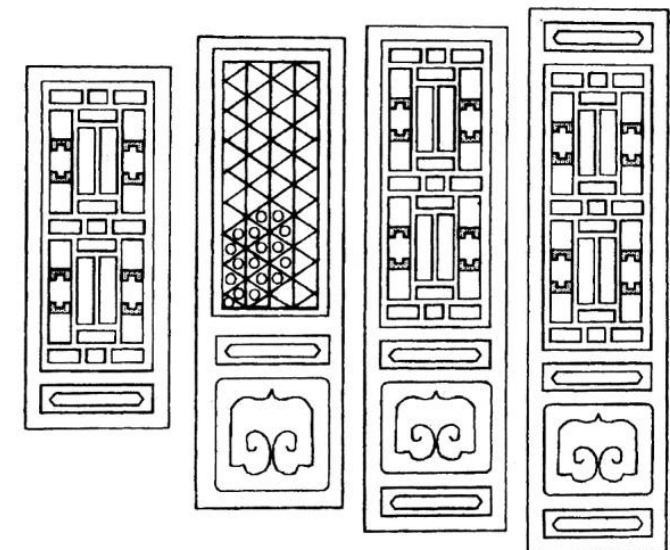

Figure 6 Variations of partition doors (drawn by the author)

\subsection{Innovation based on traditional patterns}

Traditional patterns simplified or subject to abstract artistic processing, such as the square lattice style of ceilings similar to chessboards and partitions with simple window lattice patterns, are more appropriate for use in contemporary design and are compatible with the simple modern style. While using such elements, it is necessary to explore the source of their formation, pay attention to retaining some of the essential characteristics of traditional patterns and turn them into new cultural expression symbols to manifest the continuation of historical context. For example, lattice windows in traditional gardens, as shown in Figure 7, are often made of lath bricks or gray tiles. Attention should be paid to the size and proportion of individual rectangles in their application to the interior space. Simple scaling damages the harmonious proportion of each part in the interior space as shown in the left picture of Figure 8 . The right picture in Figure 8 is more harmonious. Therefore, the size and shape of individual rectangles are fixed elements, and their combined quantity and form are variable elements.

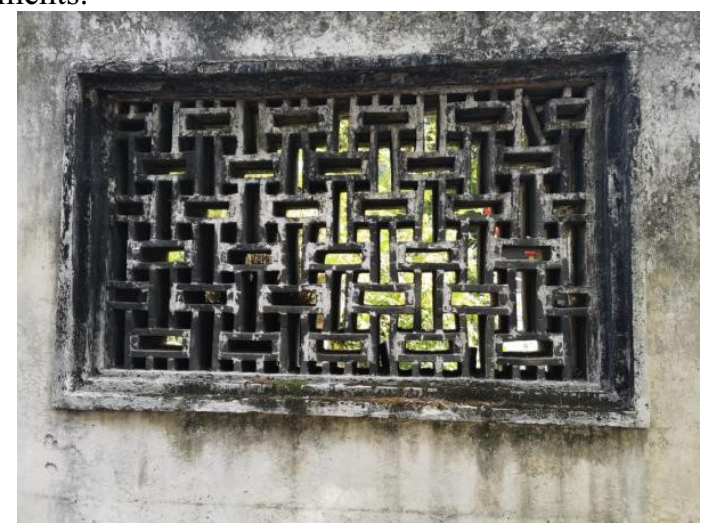

Figure 7 Lattice window in the garden of Slender West Lake 

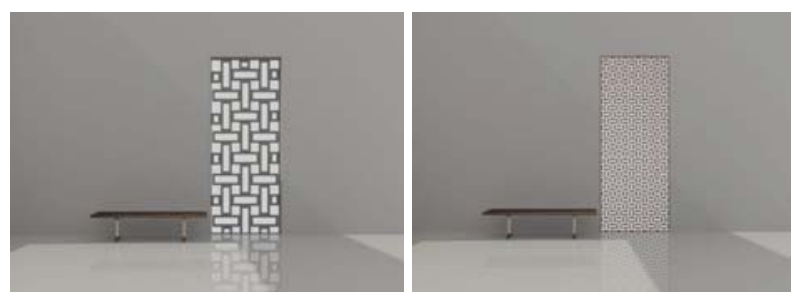

Figure 8 Indoor lattice window partition (drawn by the author)

The design of common traditional plum blossom window patterns basically follows the rule of "sparse on the top and dense at the bottom". Triangles, quadrilaterals and pentagons are dominated, with the angle not less than $15^{\circ}$ and not more than $140^{\circ}$. The left picture of Figure 9 seems incongruous and unaesthetic due to the neglect of the rule of traditional plum blossom pattern design, while the right picture is pleasing to eyes. Therefore, the rule is a fixed element and the form is a variable element.
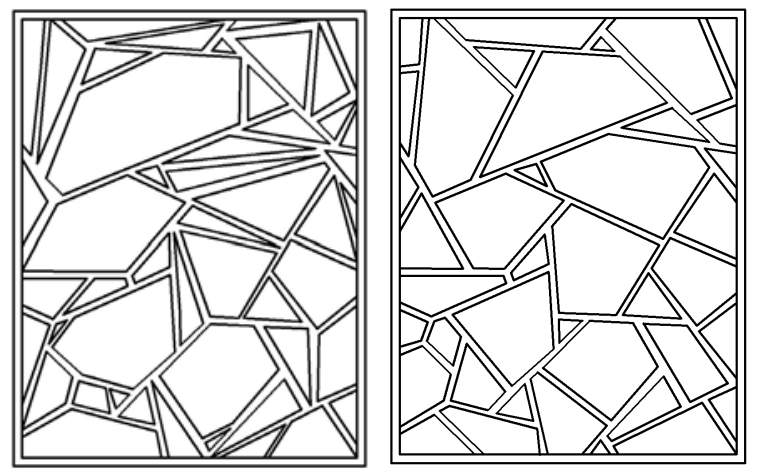

Figure 9 Patterns of ice crack windows (drawn by the author)

\section{CONCLUSION}

The introduction of new concepts and ways of thinking has provided us with more perspectives for taking a new look at the traditional culture though modern design methods have brought a lot of convenience for our interior space design. The renewal and iteration of new design representation methods also provide more possibilities for
Chinese architectural decorative design. We can only avoid the situation where the appearance is similar but the charm is insufficient by exploring the design rules of traditional architectural patterns, understanding and recreating them from the perspective of respecting traditional culture and integrating them into the interior of buildings organically.

\section{ACKNOWLEDGMENT}

This study is financially supported by 2018 Jiangsu Colleges and Universities Philosophy and Social Science Research Fund Project(2018SJA1078).

\section{REFERENCES}

1. He Yuanjun (2008), Several Issues on Computer Grraphics. Journal of Shanghai Jiao Tong University, vol. 42, No.4, p513-517.

2. Li Gang, and Xu Ren-ping (2007), Design in Geometry-Quadratic-Equation, Journal of Chongqing Jiangzhu University, vol. 29, No.5, p4953.

3. Liang Sicheng, and Ling Zhu (Ed.). Chinese Architecture: Art and Artifacts. Beijing: Foreign Language Teaching and Research Press, 2011, p213215.

4. Sun Dazhang (Ed.)2002. History of Chinese Ancient Architecture, vol. 29, Qing architecture. China Construction Industry Press, Beijing, p453-459.

5. Yi Dan, and Wang Shali (2006). The Use of Traditional Decorative Grain Type in Chinese Architecture, Furniture and Interior Decoration, No. 03.

6. Sun Xiaoqian (2015). Brief Discussion on the Application of Chinese Traditional Decorative Patterns in Modern Interior Space Design. Grain in Ear, No.15, p151-152. 